\title{
Towards Automatic Detection of Local Bearing Defects in Rotating Machines *
}

\author{
Stefan Ericsson ${ }^{\text {a }}$, Niklas Grip, ${ }^{\mathrm{a}, *, 1}$, Elin Johansson ${ }^{\mathrm{a}}$, \\ Lars-Erik Persson $^{a}$, Ronny Sjöberg ${ }^{b}$ and Jan-Olov Strömberg ${ }^{c}$

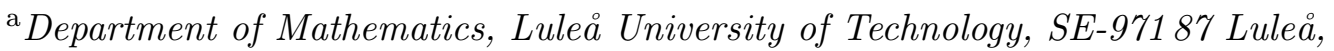 \\ Sweden.E-mail: \{sen,grip,elin,larserik\}@sm.luth.se \\ ${ }^{\mathrm{b}}$ Naiiden Teknik AB, Aurorum 30, SE-97775 Luleå, Sweden. E-mail: \\ Ronny.Sjoberg@naiden.se \\ ${ }^{\mathrm{c}}$ Department of Mathematics / NADA, Royal Institute of Technology, SE-100 44 \\ Stockholm, Sweden.E-mail: janolov@math.kth.se
}

\begin{abstract}
In this paper we derive and compare several different vibration analysis techniques for automatic detection of local defects in bearings.

Based on a signal model and a discussion on to what extent a good bearing monitoring method should trust it, we present several analysis tools for bearing condition monitoring and conclude that wavelets are especially well suited for this task. Then we describe a large-scale evaluation of several different automatic bearing monitoring methods using 103 laboratory and industrial environment test signals for which the true condition of the bearing is known from visual inspection. We describe the four best performing methods in detail (two wavelet-based, and two based on envelope and periodization techniques). In our basic implementation, without using historical data or adapting the methods to (roughly) known machine or signal parameters, the four best methods had 9-13\% error rate and are all good candidates for further fine-tuning and optimization. Especially for the wavelet-based methods, there are several potentially performance improving additions, which we finally summarize into a guiding list of suggestion.
\end{abstract}

Key words: bearing, condition monitoring, vibration analysis, signal model, prediction, classification, wavelet, Morlet, continuous wavelet transform, wavelet packets, matched filter, envelope method, periodization 1991 MSC: 93E10, 00A06, 42C40, 60G35, 62M20, 65-04, 65T60, 93-02, 93-04, 93A30, 93C $57,93 \mathrm{C} 83,93 \mathrm{C} 95$ 


\section{Introduction}

Bearing failures in rotating machines can cause both personal damages and economical loss. Manual inspections are not only expensive, but also connected with a risk of accidentally causing damages when reassembling a machine. Thus there is a clear need for non-destructive methods for predicting bearing damages early enough to wait with bearing replacements until next scheduled stop for machine maintenance. The most successful such methods in use today are all based on vibration analysis (see, e.g., [1,2]). They do, however, require special competence from the user, whereas, as the industry optimizes there is less personnel and time available for condition monitoring. Thus important information to support decisions is lost and there is a demand for more automatized and supportive bearing monitoring software.

Classical bearing monitoring methods can usually be classified as either time domain methods (see, for example, $[3,4,5,6]$ ) or frequency domain methods (see, for example, $[7,8,9,10]$ ). These methods look for periodically occurring high-frequency transients, which however is complicated by the fact that this periodicity may be suppressed. Moreover, classical Fourier methods tend to average out transient vibrations (such as those typical for defect bearings), thus making them more prone to "drown" in the background noise of harmless vibrations. A natural countermove is to use methods that show how the frequency contents of the signal changes with time. This kind of analysis is usually referred to as time-frequency analysis. The continuous wavelet transform (CWT) is one such transform which is particularly good at separating the short high-frequency outbursts of a typical localized bearing defect from long-duration low-frequency signal components (occurring, for example, at multiples of the axis rotational frequency). Since its introduction in the mideighties the theory of wavelets has grown very rapidly in almost every field of signal processing and recently research has begun in areas of mechanical vibration analysis (see, for example, $[11,12,13,14,15,16,17,18]$ ).

However, it is extremely important to point out that a new analysis technique only can provide more reliable diagnoses if the new mathematics and signal processing are combined with a deep insight into and experience of different types of rotating machinery.

This was the starting point of a unique Swedish joint research project with participation from Nåiden Teknik, the Centre of Applied Mathematics (CTM) at Luleå University of Technology, the Royal Institute of Technology (KTH)

\footnotetext{
^ Financially supported by the Swedish Institute of Applied Mathematics (ITM).

* Corresponding author. Address: Kårhusvägen 6:341, SE-977 54 Luleå, Sweden.

1 Supported by the Swedish Research Council, (postdoc fellowship no. 623-2003105) during the time when this paper was finished.
} 


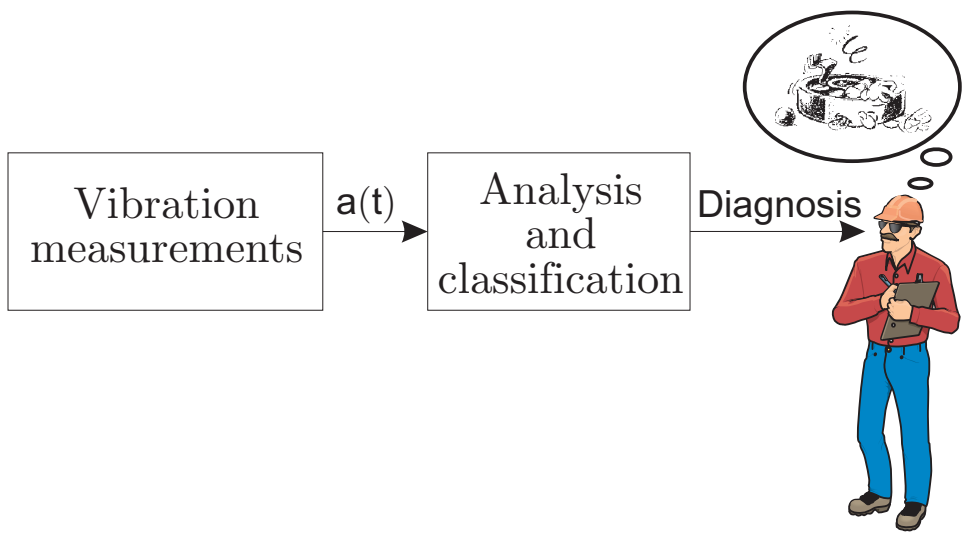

Fig. 1. The final goal is an automatic and user-friendly bering condition monitoring system.

in Stockholm, the Swedish Institute of Applied Mathematics (ITM), and the three forestry combines AssiDomän, Modo and StoraEnso. This text is a condensed and rewritten version of selected parts of the final report [19] of that project.

The final goal is an automatic bearing monitoring system with easily interpreted output data that reflects the probability of a defect bearing (see Figure 1).

We divide this analysis into three steps: First some analysis method is applied to an acceleration measurement a (here usually of length $N=16384$ ). The analyzed signal $\mathbf{b}$ requires some expert knowledge for a correct interpretation. Depending on the analysis method, the length $N^{\prime}$ of $\mathbf{b}$ is usually comparable to $N$ (or even $N^{2}$ for 2D-plots). This is too much for standard classification methods. Thus, as an intermediate step, we need to pick out the important information from $\mathbf{b}$ and reduce it to some $n$-dimensional $\mathbf{c}$ for a reasonably small $n$ (e.g., $n=2$ in plots like the one in Figure 10) (b)). Then a classification method can give the desired automatic diagnosis "functional" or "defect" (possibly with some additional judgment about the type and size of the defect):

$$
\mathbf{a} \in \mathbb{R}^{N} \stackrel{\text { Analysis }}{\longrightarrow} \mathbf{b} \in \mathbb{R}^{N^{\prime}} \stackrel{\text { Reduce dimensionality }}{\longrightarrow} \mathbf{c} \in \mathbb{R}^{n} \stackrel{\text { Classification }}{\longrightarrow \text { Diagnosis }}
$$

With the additional diagnostic power of such a method, maintenance staff will have a powerful tool and more time and concentration left for other important tasks.

In Section 2, we discuss important characteristics of and differences between different condition monitoring methods. The main focus is on the importance of a signal model and to what extent it should be trusted. Section 3 is a more detailed overview of different mathematical tools that can be combined into an 
immense number of different bearing monitoring methods. From these, we have chosen reasonable combinations, implemented them in MATLAB and compared using a large number of test signals (see Section 4) from both laboratory and industrial environments. Finally, we present our most promising results so far in Section 5.

All symbols and notation will be explained when it first appears in the text. The most frequently used notation is also collected in Table 1.

\section{The signal model and its importance}

The methods that we have considered range from methods that rely heavily on a detailed signal model to methods that work more "blindly" but without the risk of assuming too much. The former methods may perform better, but only if the model is "good enough". If the differences between model and reality are "too big" or fluctuate "too much", then a more robust method is required. We have grouped the evaluated methods into the following three categories (see also Figure 2):

\begin{tabular}{|c|l|}
\hline Notation & Meaning \\
\hline$a(t), s(t)$ etc, $t \in \mathbb{R}$ & Continuous time signals. \\
$a[k], s[k]$ etc, $k \in \mathbb{Z}$ & Sample values $(a[k]=a(k T)$ etc. $)$. \\
$\mathbf{a}, \mathbf{b}, \mathbf{c}$ etc. & Vectors. \\
$a(t)$ & Measured acceleration of vibrations $s(t)$. \\
$a_{d}$ & Decay parameter of impulse response $h$. \\
$f$ & Frequency variable. \\
$f_{0}$ & Bearing-axis resonance frequency. \\
$h(t)$ & Impulse response of the bearing-axis system. \\
$s(t)$ & Bearing vibrations $\left(s^{\prime \prime}(t)=a(t)\right)$. \\
$t$ & Time variable. \\
$A$ & Amplitude of impact oscillations. \\
CWT & Continuous Wavelet Transform. \\
FFT & Fast Fourier Transform. \\
$H(f)$ etc. & Fourier transforms: $S(f) \stackrel{\text { def }}{=} \int_{\mathbb{R}} s(t) \mathrm{e}^{i 2 \pi f t} \mathrm{~d} t$ \\
\hline
\end{tabular}

Notation used throughout the paper. All temporarily used symbols and notation are defined as they appear in the text. 
- Matched filters and Cramér-Rao estimation are examples of methods that rely hard on a rather detailed signal model.

- The largest block of methods are well established time- and frequency domain methods, as well as techniques based on time-frequency analysis. They depend less on the signal model and should therefore in general be more robust. The evaluation of these methods is complicated by the fact that there are so many tools to play with. It is not possible to evaluate all possible (combinations of) methods and all more or less important choices associated with each method, such as the choice of wavelet, thresholds, what frequencies to investigate etc. Therefore, one must restrict to comparing a reasonably small number of methods that seem likely to perform well.

- Similar to the first two mentioned methods, feature extraction is in a certain sense an optimal way to detect bearing faults, but now in the case when no reliable signal model is available. Instead, these methods are trained on test signals of all types that the methods shall be able to tell apart. Thus many test signals are needed and they cannot be created artificially (since that would require a reliable signal model and if one exists, it is our strong belief that one can achieve better performance with a bearing monitoring

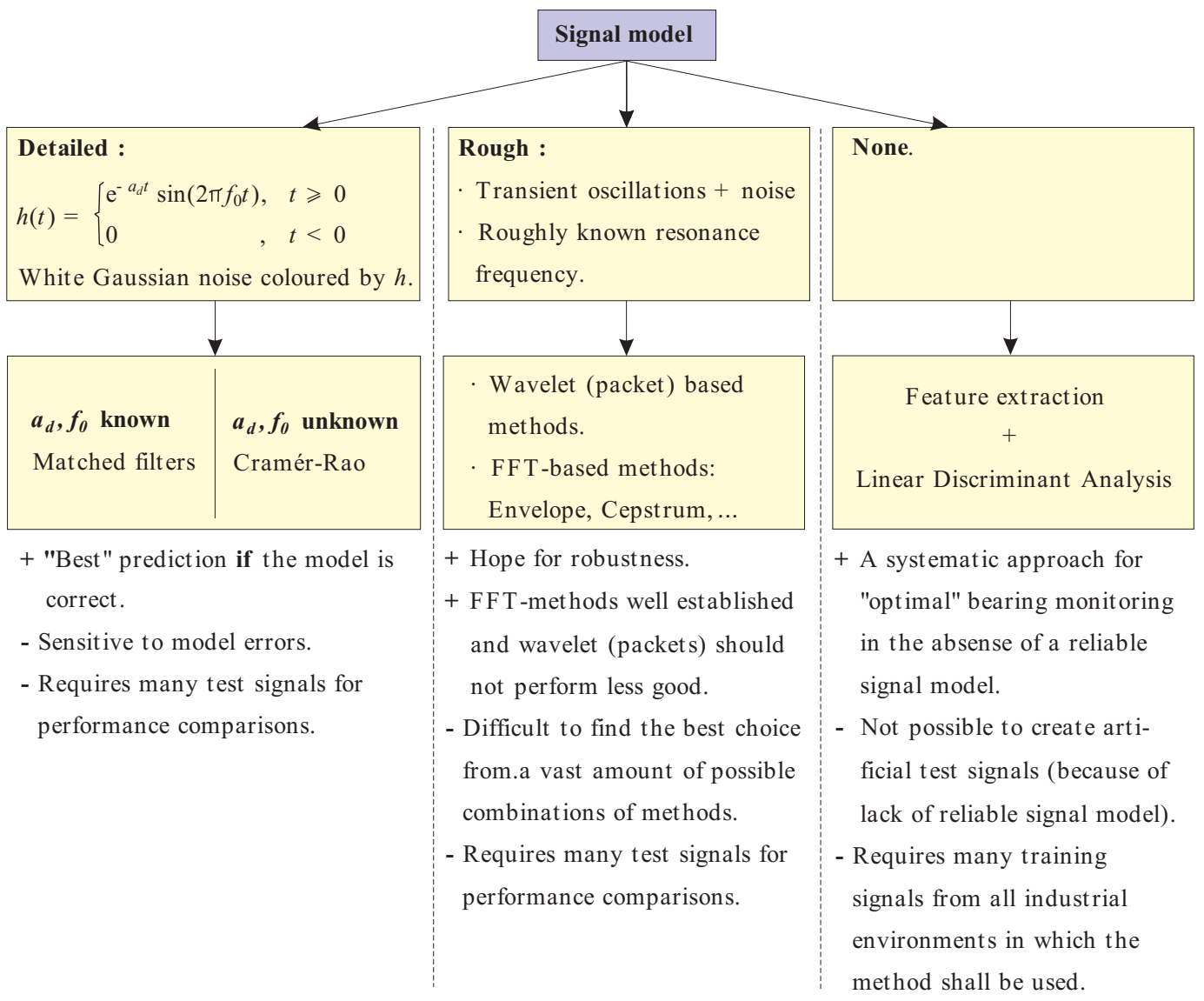

Fig. 2. Overview and classification of bearing monitoring methods. A more detailed model of bearing fault vibrations $h(t)$ and noise improve theoretical performance at the cost of larger sensitivity to model errors. 
method based on this model).

Exact knowledge of the impulse response $h(t)$ would decrease the complexity and improve the performance of several methods presented in this report. Although we have special (and not too expensive) measure equipment for measuring $h$, this equipment does require some special competence from the user. Thus it is unrealistic to assume $h$ to be exactly known in a typical industrial environment, where these measurements has to be done at some 2000 different measure points. We are therefore limited to theoretical models.

\subsection{A rudimentary signal model}

We will now propose a rudimentary mathematical model for what we mean is the most important vibration measurement characteristics that a good bearing monitoring method must recognize.

An impact impulse is generated every time a ball (or roller) hits a defect in the raceway or every time a defect in a ball hits the raceway. Each such impulse excites a short transient vibration in the bearings at its natural frequencies. The lowest such frequency, $f_{0}$, is a rigid body mode (individual balls acts as springs, see [20, page 996]). We will often refer to $f_{0}$ as the resonance frequency of the bearing.

As in [20, Chapter 26], we model the shaft-bearing system as a viscously damped mass-spring system, but instead of applying an oscillating force, we are interested in the vibrational response of the bearing and its surroundings to the impact impulses. Each impact causes an instantaneous increase of the momentum, which can be used as initial condition for the position $h(t)$ of the point where vibration measurements are are performed. A standard solution (see, for example, [21]) gives the impulse response

$$
C h(t) \stackrel{\text { def }}{=} \begin{cases}C \mathrm{e}^{-a_{d} t} \sin \left(2 \pi f_{0} t\right) & \text { if } t>0 \\ 0 & \text { otherwise }\end{cases}
$$

where $a_{d}$ is a decay (or bandwidth) parameter.

As the shaft rotates, these vibrations will occur periodically with an impact frequency $1 / T$ (computed in (3) below). With notation $A_{p}$ for the impact impulse amplitudes and $A \stackrel{\text { def }}{=} A_{p} C$, the resulting signal is

$$
s(t)=A \sum_{n} h(t-n T) .
$$

Since $s$ is the convolution of $A h$ with a $T$-periodic sequence of Dirac delta- 
distributions, $p_{T} \stackrel{\text { def }}{=} \sum_{n} \delta(\cdot-n T)$, its Fourier transform is

$$
S(f)=A H(f) P_{T}(f)=\frac{A}{T} H(f) \sum_{n} \delta\left(f-\frac{n}{T}\right) .
$$

Both $s$ and $\widehat{s}$ are sketched in Figure 3 (where the Dirac impulses are denoted with vertical arrows, showing up as well-localized sharp impulses in real applications). There, the largest peaks appear around frequency $f_{0}$, but in real measurements this frequency localization can be displaced towards lower frequencies as the bearing defect grows bigger. This would not happen if only the impact amplitude $A_{p}$ was growing. Instead we interpret this as a sign that for large defects, $p_{T}$ is sometimes better modeled as a train of rectangles

$$
p_{T}(t)=A_{p} \sum_{n=-\infty}^{\infty} r_{\varepsilon}(t-n T), \quad r_{\varepsilon}(t)= \begin{cases}\frac{1}{2 \varepsilon} & \text { if }|t|<\varepsilon \text { for some integer } \mathrm{n} \\ 0 & \text { otherwise. }\end{cases}
$$

Hence, with $*$ denoting convolution, this change of $p_{T}$ will replace the vibrations $s$ with $s_{\varepsilon} \stackrel{\text { def }}{=} A p_{T} * h$ such that

$$
\begin{aligned}
s_{\varepsilon}(t) & =A \sum_{n=-\infty}^{\infty} \int_{-\infty}^{\infty} r_{\varepsilon}(t-\tau-n T) h(\tau) \mathrm{d} \tau=A \sum_{n=-\infty}^{\infty} \int_{-\infty}^{\infty} r_{\varepsilon}(-\tau) h(t-n T+\tau) \mathrm{d} \tau \\
& =\frac{A}{2 \varepsilon} \int_{-\varepsilon}^{\varepsilon} \sum_{n=-\infty}^{\infty} h(t-n T+\tau) \mathrm{d} \tau=\frac{1}{2 \varepsilon} \int_{-\varepsilon}^{\varepsilon} s(t+\tau) \mathrm{d} \tau .
\end{aligned}
$$

Hence, $s$ is a smeared out local average $s_{\varepsilon}=s * r_{\varepsilon}$ of $s$ with Fourier transform

$$
S_{\varepsilon}(f)=S(f) R_{\varepsilon}(f)=S(f) \frac{\sin (2 \pi f \varepsilon)}{2 \pi f \varepsilon},
$$

where $\left|\frac{\sin (2 \pi f \varepsilon)}{2 \pi f \varepsilon}\right|$ decays as $1 /|f|$ when $|f| \rightarrow \infty$. This means, roughly, that the dominating parts of $S$ are displaced from $f_{0}$ towards lower frequencies.

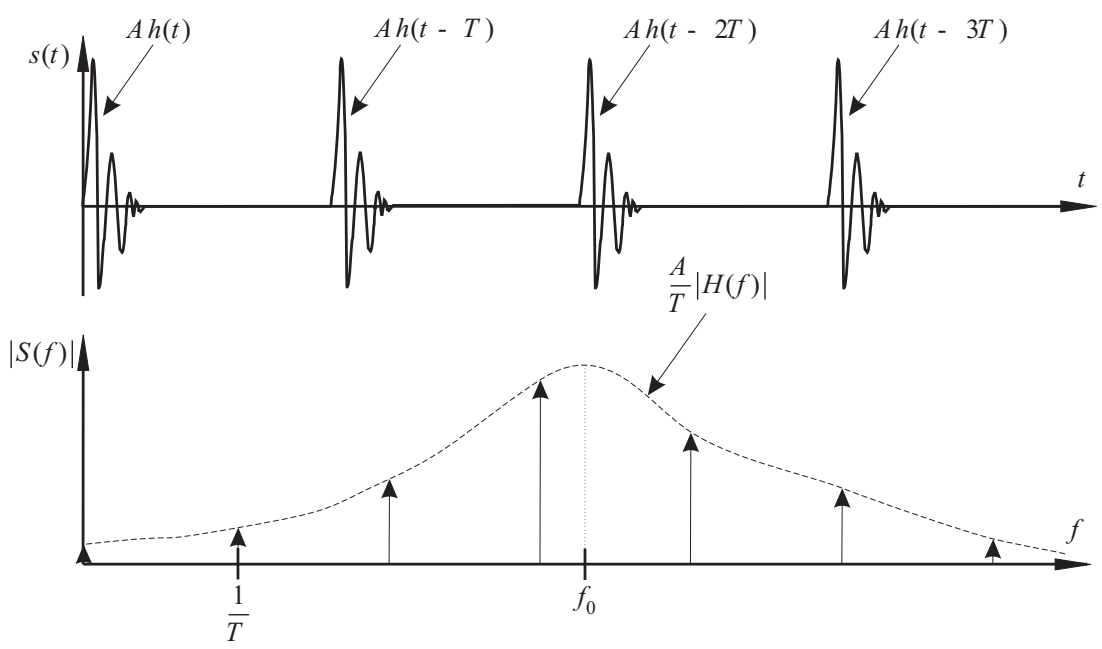

Fig. 3. Sketch of vibrations caused by a typical localized bearing defect. 
Equations (1) and (2) suggest that a good bearing monitoring method should be designed to look for transient oscillations of frequency $f_{0}$ and check whether these oscillations occur periodically with a period $T$ (note however, that it should not depend too heavily on this periodicity, as explained in Section 3). The location of a defect can then be identified if $1 / T$ coincides with one of the following frequencies, which are computed from the geometry of the bearing (see [20, Chapter 8] for details):

$$
\text { Cage frequency: } f_{\mathrm{C}}=\frac{f_{\mathrm{A}}}{2}\left[1-\frac{D_{\mathrm{b}}}{D_{\mathrm{p}}} \cos (\varphi)\right] \text {. }
$$

Outer race frequency: $f_{\mathrm{O}}=\frac{1}{T_{\mathrm{O}}}=N_{\mathrm{b}} \cdot f_{\mathrm{C}}$.

$$
\text { Inner race frequency: } \quad f_{\mathrm{I}}=\frac{1}{T_{\mathrm{I}}}=N_{\mathrm{b}}\left(f_{\mathrm{A}}-f_{\mathrm{C}}\right) \text {. }
$$

Roller (or ball) spin frequency: $f_{\mathrm{R}}=\frac{1}{T_{\mathrm{R}}}=\frac{D_{\mathrm{p}}}{2 D_{\mathrm{b}}} f_{\mathrm{A}}\left[1-\left(\frac{D_{\mathrm{b}}}{D_{\mathrm{p}}}\right)^{2} \cos ^{2}(\varphi)\right]$.

Here we used the following notation:

$$
\begin{aligned}
f_{\mathrm{A}} & =\text { revolutions per second of inner race, } \\
D_{\mathrm{b}} & =\text { ball diameter } \\
N_{\mathrm{b}} & =\text { number of balls } \\
D_{\mathrm{p}} & =\text { pitch circle diameter and } \\
\varphi & =\text { contact angle. }
\end{aligned}
$$

These formulas are theoretical and the difference between calculated and measured bearing frequencies can be as much as several Hertz. These discrepancies arise when bearings have significant thrust loads and internal preloads. This changes the contact angle and causes the outer race frequency to be higher than calculated (see [2, page 150]).

\subsection{Loaded bearings}

Only the model (2) for $s$, is used in simulations and methods described in the remaining paper. For loaded bearings with inner race or rolling element faults, the following refinements can be useful.

For a loaded bearing, the impact impulses can be written

$$
p(t)=\sum_{l=-\infty}^{\infty} A_{p} \beta(\tau+l T) \delta(t-\tau-l T)
$$


with nonzero amplitude $A_{p}$ if a defect bearing is present. The delay $\tau$ depends on where on the ball or raceway the defect is located. For an outer ring fault, $\beta(t)=1$. For an inner race fault, $\beta$ describes how the strength of the impulses varies when the defect moves into and out from the load zone. For radial load, Harris [20, pages 234-236] suggests the model ${ }^{2}$

$$
\beta(t)=\max \left(1-\frac{\varepsilon}{2}\left(1-\cos \left(2 \pi f_{A} t-b\right)\right), 0\right)^{\alpha},
$$

where $\varepsilon>2$ for a bearing with positive clearance and $\alpha$ is $3 / 2$ for ball bearings and 10/9 for roller bearings. (This model can more or less be found in [3,7].) A bigger exponent $\alpha$ gives a more pointed envelope $\beta$. The plot in Figure 4(a) illustrates how $\varepsilon$ and $b$ affect the shape and translation of $\beta$.

In this survey, we will only consider inner and outer ring defects. For detection of rolling element faults we suggest to add a factor $(-1)^{l}$, which reflects the fact that every second rolling element impact hits the inner ring and every second impact hits the outer ring (see Figure $4(\mathrm{~b})$ ), that is, to replace $\beta(t)$ in (4) with

$$
\beta_{l}(t) \stackrel{\text { def }}{=}(-1)^{l} \max \left(1-\frac{\varepsilon}{2}\left(1-\cos \left(2 \pi f_{C} t-b\right)\right), 0\right)^{\alpha} .
$$

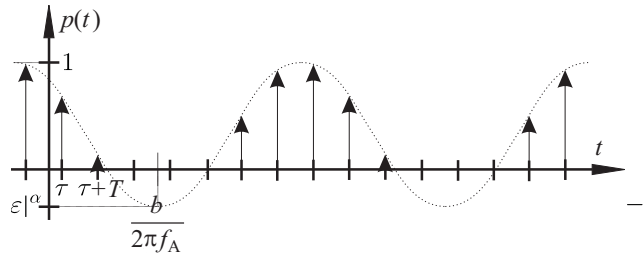

(a)

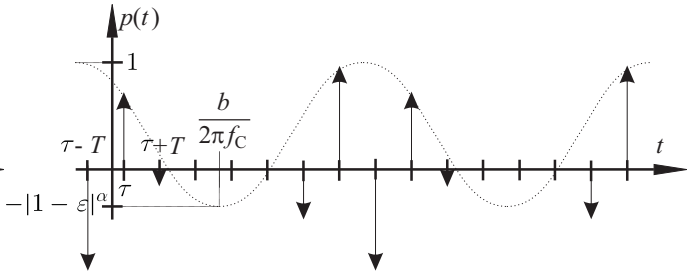

(b)

Fig. 4. Example plot of the impact impulses $p(t)$ in the case of an inner ring fault (a) and an rolling element fault (b). Each Dirac impulse $A_{p} \beta(\tau+l T) \delta(t-\tau-l T)$ in (4) is drawn as a vertical arrow with length equal to the impulse amplitude. The positive part of the dotted "envelopes" are the functions $\beta$ and $\beta_{l}$ in (5).

\subsection{Adding noise and discretizing}

It remains to adapt our model for continuous-time position-measurements (2) to the actual bandpass filtered and noisy acceleration-measurements treated in the remaining paper. We base this model on the underlying assumption that all other vibrations that the shaft-bearing system is exposed to add up

$\overline{2}$ Our $\varepsilon$ correspond to $1 / \varepsilon$ in [20], because then the case of no load simply corresponds to setting $\varepsilon=0$ in computer simulations. 


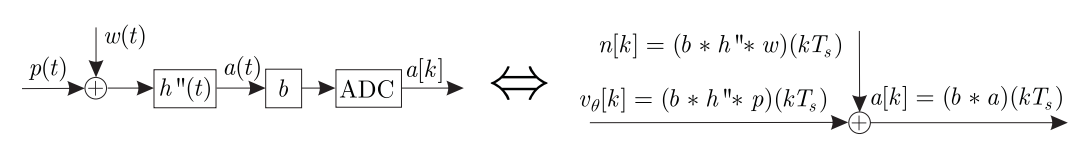

(a)

(b)

Fig. 5. The continuous time (a) and equivalent discrete time (b) system models. The sampling is performed by a bandpass filter $b$ and an analog-to-digital converter (ADC).

to zero-mean white Gaussian noise $w$. This is in no way obvious but seems reasonable and a basic comparison of model and reality in Section 2.4 gives some support for this assumption. With $*$ denoting convolution, (4) gives the resulting measured acceleration

$$
a(t)=\frac{d^{2}}{d t^{2}}((p+w) * h)(t)=\sum_{l=-\infty}^{\infty} A \beta(\tau+l T) h^{\prime \prime}(t-\tau-l T)+\left(w * h^{\prime \prime}\right)(t)
$$

Thus, we get the model depicted in Figure 5(a) and, after lowpass filtering and sampling, the equivalent discrete-time model in Figure 5(b) (for more details, see [19, Appendix C] and [22]).

\subsection{Model verification and suggested refinements}

Contrary to our assumption in Section 2.3, suppose that the sum of vibrations from different parts of a "typical" machine adds up to white Gaussian noise after the convolution with $h^{\prime \prime}$. This would remove what we think of as the main complication of bearing condition monitoring (see Section 3.4), namely that and both the signal and the noise are "coloured" by the same filter $h^{\prime}$.

In Figures 6 and 7 we compare our signal and noise model with real measurements. For a simple but illuminating visual comparison, we plot the absolute value of the continuous wavelet transform (CWT) of the compared signals (as described in Section 3.3). Figure 6 shows a clear difference (in smoothness and frequency localization) between bandpass filtered white Gaussian noise (the lowermost plot) and the test rig vibration measurements from a functional bearing in the topmost plot. This plot looks more like the topmost plot in Figure 7, which shows Gaussian noise colored according to our model. The other plots in that figure show how increasing signal-to-noise ratio (SNR) gradually transforms the CWT to one more similar to the CWT of vibrations caused by a big outer race defect shown in the middle plot of Figure 6. Still, there are some differences in the exact shapes of the bumps and fast Fourier transform (FFT) plots of some test signals also show deviations from our model at low and high frequencies. Thus, methods that rely much on a precise signal model may require further model refinements for good performance. Based on the above observations, we give a few suggestions for improving model 
correctness:

- To use a more detailed model of $h$ and/or to adjust bandpass filters to (if possible) include possible resonance frequencies but block frequency bands where model deviations are known or believed to exist.

- Additional white or colored noise at low frequencies. Perhaps colored by other parts of the measurement environment (e.g., there is often disturbing vibrations like vibrations oscillating with multiples of the axis rotational frequency or twice the motor feeding frequency).

- Other natural frequencies of the bearing-axis system [20, page 996] may be dominating the signal at higher frequencies.

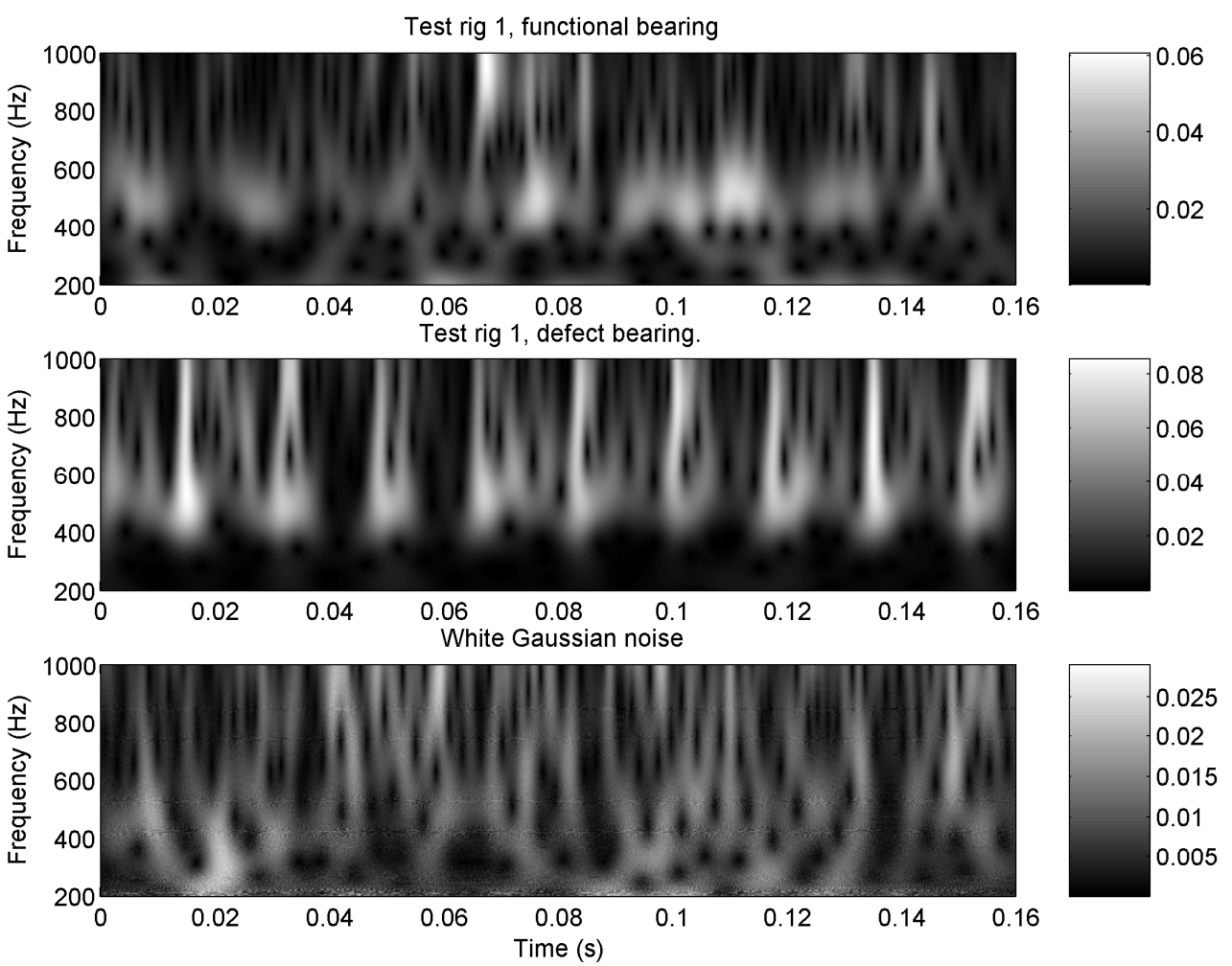

Fig. 6. The topmost two plots show continuous wavelet transforms (CWT) of test rig measurements. The clear difference from the lowermost CWT of white Gaussian noise (bandpass filtered as described in Section 4) confirms that the noise not is white and Gaussian. Instead, the topmost two plots show more resemblance with corresponding plots in Figure 7, which is computed from our signal and noise model. 


\section{Choice of basic methods}

Underlying our choice of bearing monitoring methods to evaluate, are the following observation and restrictions:

- As described in Section 2.2 (and observed in some of our test signals), the load zone dependence of inner race bearing defects can suppress the periodicity of the bearing impacts. This makes such defects more difficult to find with a method that depends on this periodicity (such as the Fourier methods in Section 3.2) whereas the performance of methods that only reacts on a single (large enough) impact would be left unaffected. Still, this is no motivation for ignoring the extra information contained in the (possibly suppressed) impact periodicity.

On the contrary, in Section 3.4 we will see from matched filter theory that it is possible to obtain maximum signal-to-noise ratio for the bearing impacts by convoluting with the "inverse" of the impulse response $h$ and look for the original impact impulses in white Gaussian noise, which by nature already contains large but rare impulses. Thus a single impact can be interpreted as a probable bearing defect only if it has very large amplitude

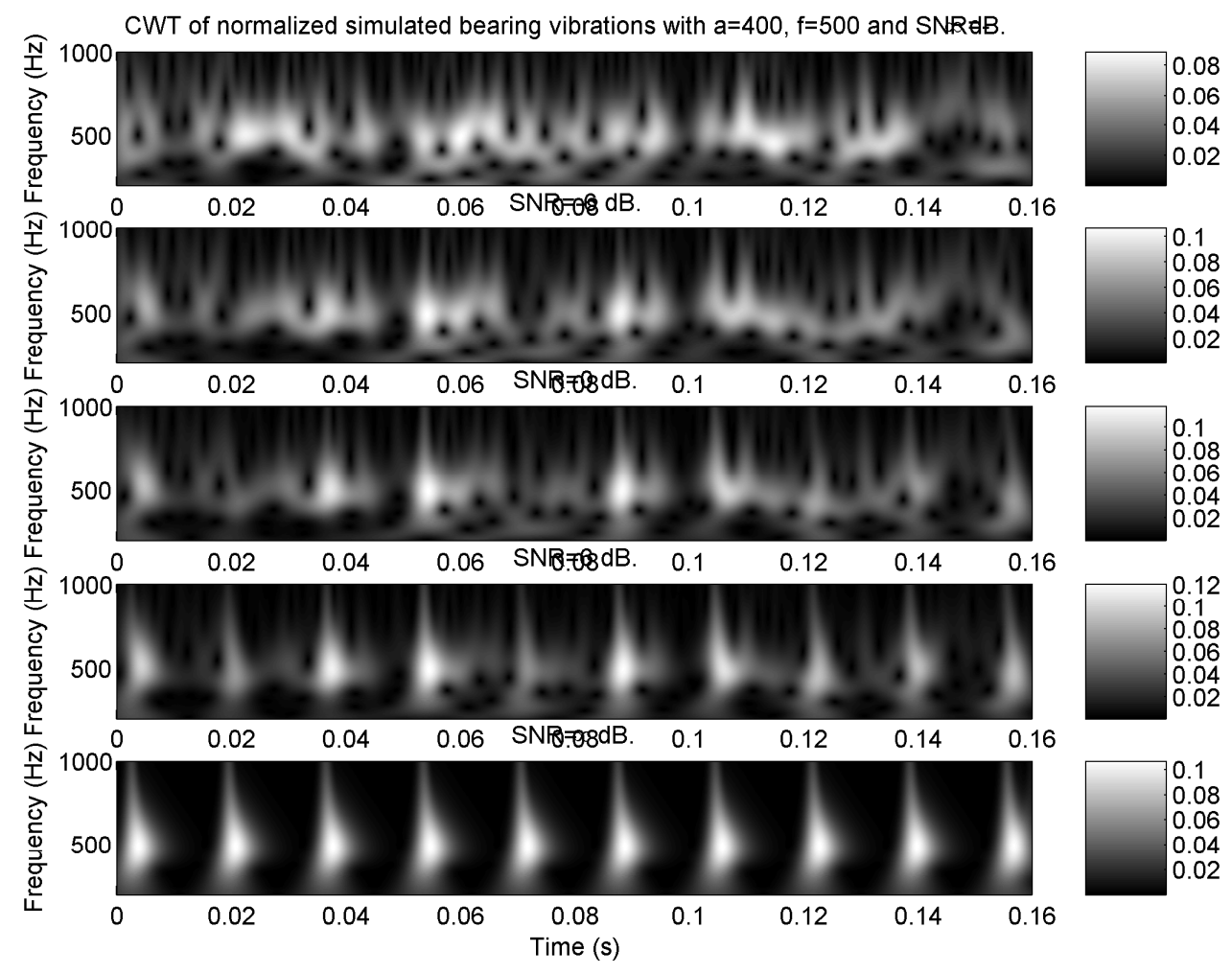

Fig. 7. CWTs of simulated bandpass filtered bearing impacts with different signal-to-noise ratios. 
(relative to the standard deviation of the noise), so that it is very unlikely that it is just a natural part of the white noise. For impulses that repeat with a (possibly suppressed) frequency coinciding with the inner ring impact frequency, much smaller impulse amplitudes are needed for detecting an equally probable inner ring defect. This is also the reason why, in Figure 6, some minor "bumps" are visible at the resonance frequency also in the first plot. The important difference is that in the second plot one can conclude from the amplitude and periodicity of the bumps that they originate from a large outer ring defect. Thus it is our strong opinion that although it is desirable for a bearing monitor method to be able to detect a single (and large enough) impact, an optimal (in any reasonable sense) method must also be able to detect impact periodicity (suppressed or not) of possibly smaller impacts.

- Another important matter is whether old measurements are available or not. In this text we aim for reasonably good performance without comparing with old measurements. Then the performance of all considered methods will surely improve with time, when there are old measurements to compare with.

- Similarly, although the actual vibration amplitude can be significantly different depending on the kind of bearing and environment, the amplitude does bring important information if it can be compared to the amplitude of some well-known signal component or some computed threshold reference value, but the following methods analyze only the shape of the signal (most methods will be homogeneous and this even includes nonlinear thresholding for certain threshold functions). So to assure a fair comparison of methods, all test signals are normalized to $L_{2}$-norm $\|s\|_{2}=\sqrt{\sum_{n}\left|s_{n}\right|^{2}}=1$. Once again, in situations where we have something to compare with, the original norm can be used to improve the performance of all tested methods.

Based on these restrictions, we have chosen to evaluate methods that are based on the following basic tools (described in more detail in the original report [19]). The evaluation results for the so far best performing such methods then follow in Section 5.

\subsection{Time methods}

Curtosis and crest factor: Some common "peakiness" estimates of a signal $s$ are the Kurtosis factor $\|s-\bar{s}\|_{4}^{4} /\|s-\bar{s}\|_{2}^{2}$ (where $\bar{s}$ denotes the mean of $s$ and $\|x\|_{n}^{n} \stackrel{\text { def }}{=} \sum_{k}\left|x_{k}\right|^{n}$ ), the crest factor $\max _{k}\left|s_{k}\right| /\|s\|_{2}$ (see, e.g., [3,5]) and other variations on the same the same theme. 
Autocorrelation and cepstrum: The autocorrelation of $s[k]$ is defined by

$$
a_{s}[n] \stackrel{\text { def }}{=} \frac{1}{2 N+1} \sum_{n=-N}^{N} s[k] s[k-n]^{*}
$$

with * denoting complex conjugate. With uppercase notation for the discrete Fourier transform $S[k]=\frac{1}{\sqrt{2 N+1}} \sum_{n=-N}^{N} s[n] \mathrm{e}^{-i \frac{2 \pi k}{2 N+1} n}$ it follows from the Parseval relation and the $2 N+1$-periodicity of $S[k]$ that

$$
\begin{aligned}
& a_{s}[n]=\frac{1}{2 N+1} \sum_{k=-N}^{N} s[k] s[k-n]^{*}=\frac{1}{2 N+1} \sum_{k=-N}^{N}|S[k]|^{2} \mathrm{e}^{i \frac{2 \pi k}{2 N+1} n}, \\
& A_{s}[k]=\frac{1}{\sqrt{2 N+1}}|S[k]|^{2}, \quad k=-N,-N+1, \ldots, N,
\end{aligned}
$$

where, under standard assumptions about ergodicity and "large enough" $N,|S[k]|^{2} /(2 N+1)$ is the power spectrum of $s$ (see, e.g., [23,22]). A related mathematical tool is the cepstrum $c_{s}$ defined via its discrete Fourier transform

$$
C_{s}[k]=\log |S[k]|^{2}, \quad k=-N,-N+1, \ldots, N
$$

and described in more detail by Randall [24]. He argues that the power spectrum of a faulty ball bearing may contain small scale (compared to the largest peaks in the spectrum) periodic structures that can appear clearly in $c_{s}$ but not in $a_{s}$. Periodicities in $s$, with period $T$, will show as peaks in $a_{s}$ and $c_{s}$ at integer multiples of $T$.

Periodization: Another tool for making 1/T-periodic impacts more visible is the transformation

$$
\left.s \longmapsto p(s) \stackrel{\text { def }}{=} \frac{1}{N \max |s|} \sum_{n=0}^{N-1} s(\cdot+n T)\right|_{[0, T)} .
$$

The function $p$ emphasizes parts of $s$ that are periodic with repetition frequency $1 / T$. Since the outbursts we are searching for have rather high oscillation frequency, the estimated value of $1 / T$ has to be quite accurate. Note, for a simple example, that $\cos \left(10^{17} x\right)+\cos \left(10^{17}(x+T)\right)$ is very sensitive to small changes in $T$, whereas a demodulation of the cosines completely removes this sensitivity. Hence, we suggest to first demodulate $s$. In our implementations, we have in fact computed $p(d(b(s)))$, where $b$ is a bandpass filter that cuts out the interesting frequency range $(150-1000 \mathrm{~Hz})$ and the demodulation $d$ computes the absolute value of the analytic version of $s$. The analytic version of $s$ (also called the Hilbert transform of $s$ ) is defined as twice the inverse Fourier transform of $u(f) S(f)$, where $u$ is the unit step function.

Note 1 Our periodization function $p$ is related to synchronous time averaging (see, e.g., [2]), which uses a trigger, placed, e.g., on the axis for an exact 
synchronization of the data collection with the rotational speed of the axis. Usually the average of at the very least 100 measurements are computed, and the method is used to "average out "frequencies that not are multiples of the rotational frequency. Synchronous averaging can therefore be useful, e.g., for finding defects in gears. For the handheld devices and industrial applications that we have in mind, at most 20 averages is possible and triggers are too time-consuming and therefore "replaced" by deconvolution.

\subsection{Frequency methods}

Power spectrum: The classical use of the Fourier transform is to search for the periodically repeated peaks in the power spectrum shown in Figure 3 (also described, e.g., in $[24,1,2]$ ).

Envelope method: The resonance frequency oscillation of the impacts and the possibly suppressed impact periodicity are two modulations of the vibrations that both reduce the amplitude of the power spectrum peaks, which therefore are more likely to be suppressed below the overall noise level. A popular countermove is to remove the resonance frequency modulation with the envelope method, which consists of a bandpass filter (including the resonance frequency) followed by a demodulation and a fast Fourier transformation (see Figure 8 and for complementary details, e.g., [24]).

Both these methods use a bandpass filter to focus on a range of frequencies which must be wide enough to include the (roughly known) resonance frequency. Thus it is likely that also oscillation frequencies where bearing impact oscillations not are dominating are included in the analysis, with consequences such as lower signal-to-noise ratio and more sensitivity to possible suppressions of the impact periodicity.

\subsection{Time-frequency methods}

Time-frequency analysis provides tools for a more systematic bandpass filtering at a whole range of possible oscillation frequencies with "optimally sharp" bandpass filters (the Morlet wavelet below). This makes it possible to combine the good properties of frequency methods with an automatic search for exactly

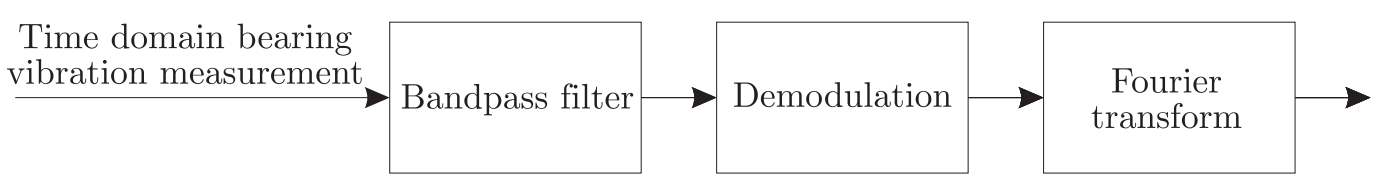

Fig. 8. A block scheme for the envelope method. In our implementations, we have used the absolute value of the Hilbert transform for demodulation. 
the oscillation frequencies (if any) where periodically repeated transients can be most clearly detected. Due to the resulting higher signal-to-noise ratio, we also expect such methods to be less sensitive to suppressions of the impact periodicity.

Continuous wavelet transform (CWT): We have chosen to use the CWT for time-frequency analysis, because it is good at separating transient highfrequency outbursts (such as bearing impacts) from the long-duration lowfrequency vibrations typically appearing around, for example, the axis rotational frequency. For optimal simultaneous time- and frequency resolution (in the sense of minimal Heisenberg box area), we use a Morlet wavelet. For more about both this and a brief introduction to time-frequency analysis in general, see, e.g., [25, Chapter 1] or [26, Chapter 1].

In plots of the CWT amplitude, (such as Figure 6), "large enough" bearing impact vibrations appear as periodically occurring "bumps", visible for the human eye. As described in the beginning of this section, due to the coloured noise, the difference between smaller defects and functional bearings is more difficult to see from visual inspection of a CWT plot, so a more systematic analysis is required for telling these cases apart. We propose and evaluate two such methods in Section 5.3.

The CWT and wavelet packets (discussed below) are the computationally most expensive tools discussed here, but since the analysis is to be done on a separate personal computer and not by the measuring device, this complexity is not a problem.

Discrete wavelet transform (DWT): For a certain class of wavelets, all information about the original signal is contained in only a discrete set of points of the CWT. These point values can be computed very fast with the DWT. It lacks the optimal simultaneous time- and frequency resolution of the Morlet CWT and is not intended for visual inspection, but is a very powerful tool for, for example, noise reduction. One way to do this is to modify the wavelet coefficients using (hard or soft) threshold functions (see, e.g., $[27,28]$ for more details) and then compute the inverse DWT. There is much work behind the "correct" choice of threshold function. Several algorithms are proposed in the literature. However, most of them assume Gaussian white noise. Noise reduction is also a natural first step in virtually any other analysis method.

Wavelet packets: The wavelet packet transform is a generalization of the DWT that, in combination with a suitable chosen cost function for the best basis algorithm (see, e.g., [28] for details) also can be very useful for bearing condition monitoring. However, it requires a lot of work to find a suitable cost function that enhances bearing faults.

Gabor and Wigner-Ville transforms: These transforms can be used as the continuous wavelet transform and at least the Gabor transform has a relatively fast discrete version. (See, e.g., [29,28].) 


\subsection{Statistical methods}

Matched filters: With notation as in Figure 5 but without the bandpass filter $b$, we can easily compute the actual vibrations $s$ from their measured second derivative $a$ (by integration or, as a simple approximation in practical applications, with cumulative sums). Hence if $h$ has strictly nonzero Fourier transform $H$, then there exists a filter $m, M(f)=1 / H(f)$, such that the original impacts and white Gaussian noise can be reconstructed with the convolution $p+w=m * s$. In fact, for our model, with $h$ given in (1), all this is possible and a straightforward computation gives a simple formula for $m$ :

$$
m(t)=\frac{1}{2 \pi f_{0}}\left(\left(a_{d}^{2}+4 \pi^{2} f_{0}^{2}\right) \delta(t)+2 a_{d} \delta^{\prime}(t)+\delta^{\prime \prime}(t)\right),
$$

where $\delta$ is the Dirac delta distribution. It follows from matched filter theory (see, e.g., [23]), that the filter $m$ is optimal in the sense that it maximizes the signal-to-noise ratio (SNR). Hence, for maximal SNR, (8) shows that one should analyze the signal

$$
p(t)+w(t)=s * m(t)=\frac{\left(a_{d}^{2}+4 \pi^{2} f_{0}^{2}\right) s(t)+2 a_{d} s^{\prime}(t)+s^{\prime \prime}(t)}{2 \pi f_{0}} .
$$

Since $s, s^{\prime}$ and $s^{\prime \prime}$ are quite similar in shape and oscillation frequency, we expect that a very precise model and parameter knowledge is required for the terms in (9) to really add up to the original impact impulses and (in a noise-free environment) vanish elsewhere.

For the actual bandpass filtered input of a practical application, a more precise computation would replace (9) with a discrete-time convolution with the Fourier series coefficients of the corresponding bandpass filtered $m(t)$.

Recall also that, $a_{d}$ and $f_{0}$ have to be guessed. Thus in a practical situation we can only hope for (the bandpass filtered version of) (9) to give nearly optimal SNR.

Since the parameters in (9) are roughly known for our test rig signals, we have applied (9) to measurements on a bearing with a very large defect (audible when the test rig is running) for a reasonably large number of possible parameter values. Still, this was not enough to result in an SNRimprovement visible for the eye. This simple test indicates a need for either a more precise signal model or a more robust analysis method, so the existence of a matched filter seems to be mainly of theoretical importance (as in the beginning of Section 3). A more detailed study would be interesting but is out of the scope of this paper.

Cramér-Rao lower bound: A minimum variance unbiased estimator $\widehat{A}$ of the bearing defect amplitude $A$ (with amplitude $A=0$ meaning "no defect") is the estimation theory name for an analysis method which, given 
a vibration measurement, computes an estimate $\widehat{A}$ of $A$, and which on average will find the correct value (that is, it is unbiased). Moreover, it is an optimal such method in the sense that the average squared error (the variance) is minimal. In our case we have a few more unknown parameters (such as $a_{d}$ and $f_{0}$ in (1)), that can be grouped into a vector $\boldsymbol{\theta}$. There is an lower bound (the Cramér-Rao lower bound) for the error covariance of any linear method for estimating $\boldsymbol{\theta}$ and a standard way for computing it, which however turned out not to be practically useful due to numerical problems in a test-implementation for our model and signals. All this and some alternative computational approaches is described in full detail in [19, Appendix D.2].

\subsection{Feature extraction}

A sampled version of the continuous wavelet transform with 128 scales containing 16384 samples each can be seen as one point in $\mathbb{C}^{2^{21}}$. The name feature extraction is used for a collection of methods for reducing the number of dimensions by mapping this point to an element in, say, $\mathbb{C}^{10}$, but without removing "too much" relevant information. Relevant here means that it still is possible to separate functional bearings from faulty ones. Feature extraction is usually combined with a classification method (corresponding to the classification lines in the plots of Section 5).

In [30] and [19, Appendix B] we describe in detail an implementation of a wavelet-based feature extractor called local discriminate bases (LDB). The first results were promising but a more full-scale evaluation would require both a large set of training data and then a large-scale test on another large set of test signals. This is out of the scope of this article.

\section{Experimental setup}

From a larger set of measurements, we have chosen 81 industrial and 22 laboratory signals for which the size of all bearing faults are known from manual inspections. For easy reference, we will use the same names on plotted signals as in [19, p. 58-64], which is a detailed descriptions of the signals, bearings machines and defects.

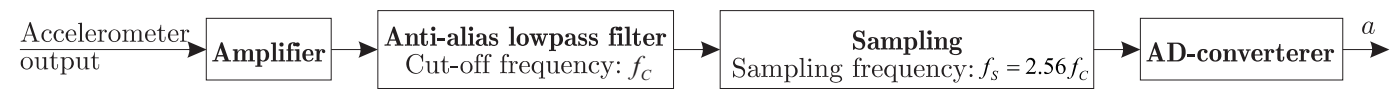

Fig. 9. A block diagram of the PerCon3 measurement device. 


\section{Collection of data}

All input data have been collected with a handheld device (a Nåiden PerCon 3 ), which record simultaneous vibration measurements in 3 orthogonal directions (horizontal, vertical and axial). An accelerometer (with sensitivity 100 $\mathrm{mV} / \mathrm{g}$ ) is magnetically attached to fixed measurement points on the machine. The accelerometer produces a charge that is proportional to the acceleration of the surface. This charge is high-pass filtered to adjust for transducer bias and then measured according to Figure 9. Finally, in all our evaluations, we also highpass filter the measurement $a$ with the cut-off frequency $200 \mathrm{~Hz}$, which is chosen so that we do not need to worry about typical low-frequency disturbers, such as the line frequency or the rotational frequency of the axis.

\section{Experimental results}

Using MATLAB and all 103 test signals, we have evaluated several different combinations of the basic methods described in Section 3. In the following subsections, we describe and present a more extensive and systematic evaluation of the four best performing methods so far. Then we summarize all experimental results in Section 5.4 and give further conclusions and suggestions for future improvements in Section 6.

The following methods were developed for another set of test signals and are therefore in no way optimized for providing good performance with the test signals at hand. In the classification plots (such as Figure 11 (b)), each test signal corresponds to one plotted point. Functional and defect bearings are denoted with dots and stars respectively. As a simple classification rule we have separated the stars from the dots with a threshold line, which can be used to classify new signals. We have chosen a line that minimizes the misclassification rate:

$$
\text { Misclassification rate }=\frac{\text { Number of points on wrong side of the line }}{\text { Total number of points }}
$$

Note, however, that the misclassification rate depends on the mixture of test signals. For example, if almost no test signal comes from a machine with a defect bearing, one would get a good misclassification rate for a "method" that classifies all bearings as functional. However, roughly half of our test signals are from machines with a defect bearing, so the misclassification ratio is a useful measure for comparing different methods. Two other performance 
measures that are important from an implementation point of view are the

$$
\text { false alarm rate }=\frac{\text { number of functional bearings detected as "defect" }}{\text { number of functional bearing test signals }}
$$

and the

$$
\text { miss rate }=\frac{\text { number of defect bearings detected as "functional" }}{\text { number of defect bearing test signals }} .
$$

If the mixture of test signals is "realistic" (for a given (type of) industrial environment), then the false alarm rate is an estimate of the probability that the method detects a defect when measuring on a functional bearing. Similarly, the miss rate is an estimate of the probability that the method fails to detect any bearing faults in a measurement from a machine with a bearing fault. There is always a trade-off between these probabilities and it depends on the type of machine and environment which of these probabilities is the most important one to minimize. Thus any method can be fine-tuned for a particular type of machine in the following way: First find the minimum misclassification rate for a (sufficiently large and realistic) set of test signals. Then adjust the classification line so that the misclassification ratio remains (close to) minimal and the proportion between false alarm rate and miss rate is satisfactory for the application at hand.

Note, finally, that some of the methods not presented here also may perform well after further refinements, especially feature extraction, which gave some very promising first results but still is not explored thoroughly enough for any final conclusions.

\subsection{The envelope method}

For each test signal and measurement direction, we have first applied an 150$10000 \mathrm{~Hz}$ bandpass filter and demodulation as in Figure 8. Then we add the resulting envelopes in $l_{2}$-sense $\left(e=\sqrt{e_{x}^{2}+e_{y}^{2}+e_{z}^{2}}\right)$ before computing the FFT. In Figure 10 (a) we plot the results for two measurements on a condensate pump. Note the clearly visible peaks at multiples of the inner ring impact frequency $120 \mathrm{~Hz}$ for the measurement on a defect bearing. These peaks are missing for the other signal, which is an identical measurement after replacing the faulty bearing.

Figures 10 (b) and (c) show the result of applying the following automatic classification to all test signals: Due to measurement precision (usually about $2 \%$ ), the impact frequencies in (3) are known only up to some maximum error $\varepsilon$. Thus, suppose that we are looking for peaks at multiples of a repetition frequency $f \pm \varepsilon$, known up to a maximum error $\varepsilon$. Then, for $n=1,2,3$, 
the $n$th peak (if any) is in the interval $I_{n} \stackrel{\text { def }}{=}[n(f-\varepsilon), n(f+\varepsilon)]$. Thus, by dividing the maximum amplitude in $I_{n}$ with the median value between the intervals $I_{n}$ and $I_{n+1}$ we get a peak-to-median ratio that we use as a measure of the size of the biggest peak in $I_{n}$. Since our measurement device has a fixed maximum signal length, an increasing sampling frequency gives higher frequency resolution, a more precise hit of peak values and, consequently, a higher peak-to-median ratio. Thus, for identical sampling frequency and a fair comparison, we upsample input signals with lower sampling rate to $25.6 \mathrm{kHz}$ (see, e.g., [22]). A noise reduction also improved the performance of the resulting algorithm:

(1) Upsampling to $25.6 \mathrm{kHz}$.

(2) Wavelet packet noise reduction using a Daubechies 9 wavelet), expanding into 9 levels, using Shannon entropy (see, e.g., [27,28]) and keeping the

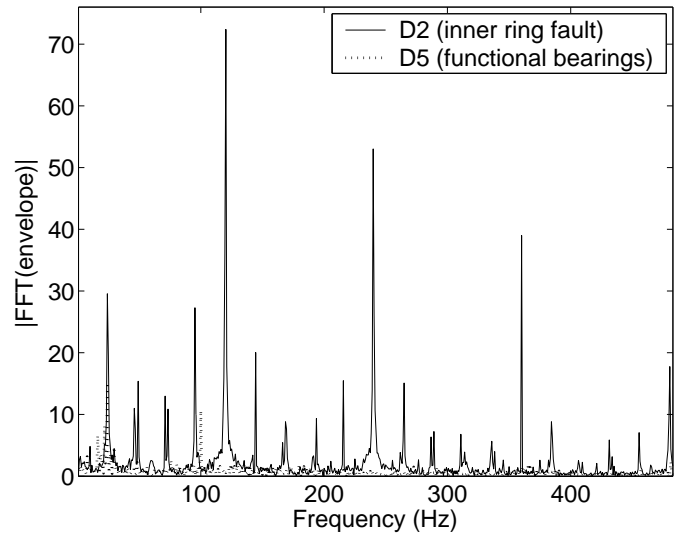

(a)

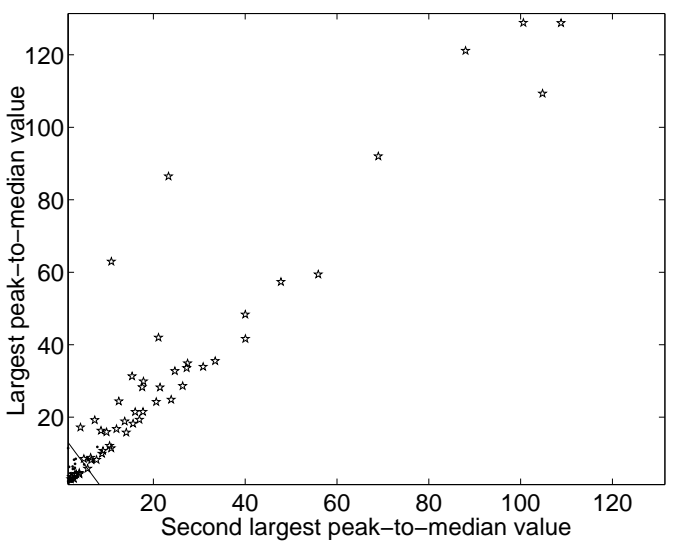

(b)

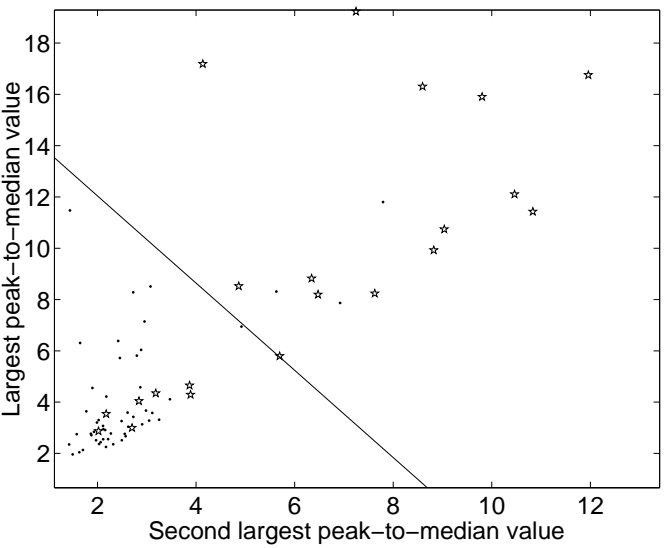

(c)

Fig. 10. The envelope method: (a) Example plot for measurements on a condensate pump before and after replacing a faulty bearing. (b) Automatic classification $(\cdot=$ functional and $\star=$ defect bearing). The misclassification rate is $10 / 103 \approx 10$ \%. (c) Part of plot (b) in close up. 
$M$ largest coefficients, with $M$ chosen to be 20 times the number of expected outbursts. (By experiments we have found that 20 coefficients is more than enough for representing one outburst.)

(3) Envelope method computed as described above.

(4) Compute peak-to-median ratios for $n=1,2,3$ and for each repetition frequency in (3). Keep the two largest and use as coordinates for one point in the plane.

\subsection{Periodization method}

In Figure 11 (a), we apply the periodization method (described in Section 3.1) to test rig measurements on bearings that are identical except for an outer ring fault on the one called J1a. In the computations for these plots, the period $T$ in the algorithm corresponds to the outer ring frequency, which gives a very clear "bump" in the plots for J1a, with maximum values $0.54,0.65$ and 0.66. Six smaller maximum values follows when repeating the same periodization computations for J1a with $1 / T$ equal to the inner ring and ball repetition frequency, respectively. Thus we choose the two largest maximum values 0.65 and 0.66 to be the coordinates of the corresponding point in Figure 11 (b). Some of our test signals are measured with an older measurement device with less memory, so for a fair comparison, we have evaluated this method only on those 89 (of 103) test signals for which we can set $N=20$ periods. Thus we end up with the following algorithm:

(1) For each repetition frequency in (3) and each measurement direction,

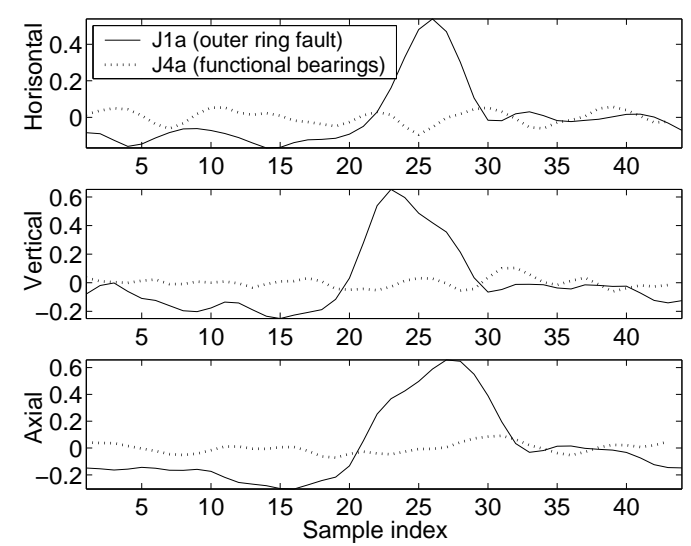

(a)

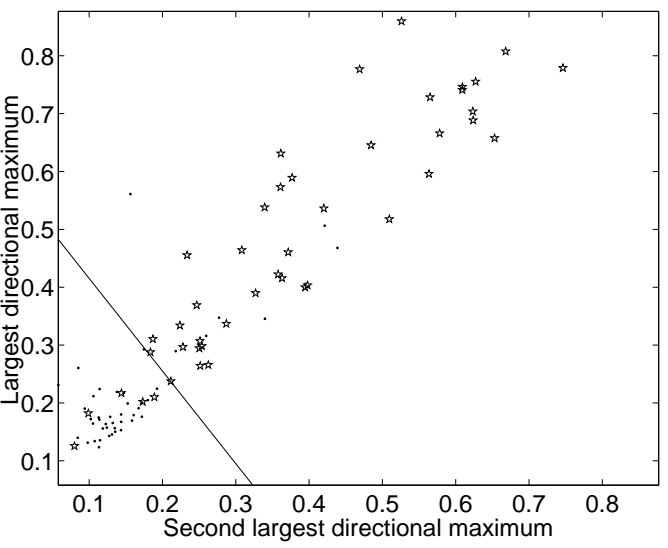

(b)

Fig. 11. The periodization method applied to test rig measurements on identical bearings with and without outer ring fault: (a) Analysis of signals measurements in horizontal, vertical, and axial direction. (b) Automatic classification $(\cdot=$ functional and $\star=$ defect bearing). The misclassification rate is $12 / 89 \approx 13 \%$. 
apply the periodization method and find the maximum value.

(2) Use the two largest maxima as coordinates for one point in the plane.

\subsection{CWT-based methods}

We describe two methods (denoted CWT1 and CWT2) that are based on the continuous wavelet transform $\mathcal{W}_{\Psi} s\left(a_{k}, t\right)$ of the analyzed signal $s$, with a Morlet wavelet $\Psi(t)=e^{i \omega_{0} t-t^{2} / 2}, \omega_{0}=5$ and with wavelet center frequencies (see, e.g., [26])

$$
\frac{\omega_{0}}{a_{k}}=20+k \frac{f_{\mathrm{s}} / 2.56-20}{100}, \quad k=0,1, \ldots, 100
$$
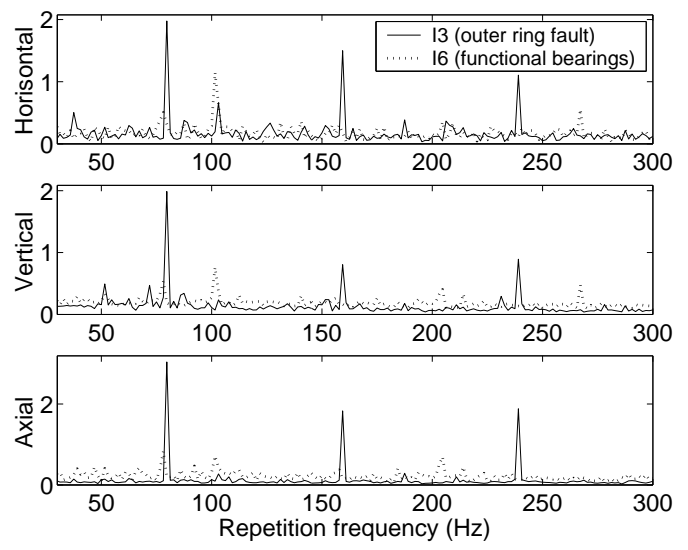

(a)

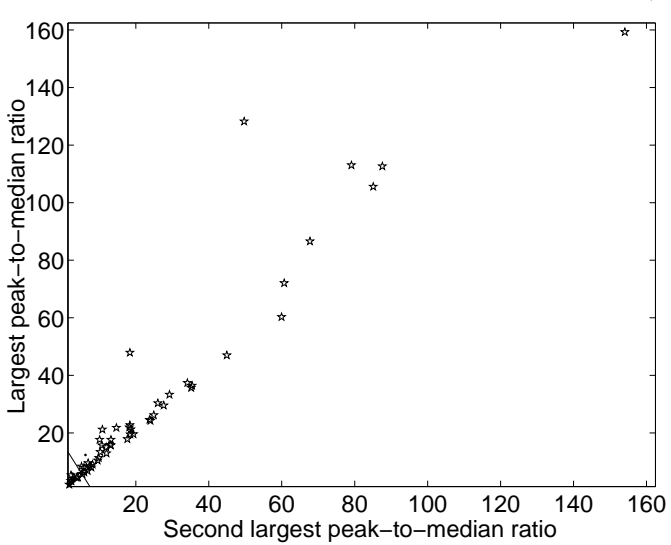

(b)

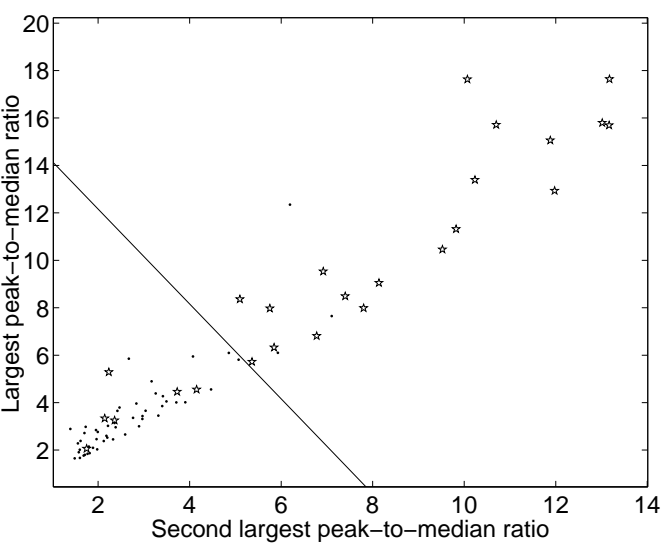

(c)

Fig. 12. The CWT1 method: (a) Example plot of $\sum_{k=0}^{100} f_{k}$ for the signals I3 and I6. (b) Automatic classification $(\cdot=$ functional and $\star=$ defect bearing). The misclassification rate is $9 / 103 \approx 8.7 \%$. (c) Part of plot (b) in close up. 
where $f_{\mathrm{s}}$ is the sampling frequency of $s$. Both algorithms analyze the functions

$$
e_{k} \stackrel{\text { def }}{=}\left|\mathrm{FFT}\left(\left|\mathcal{W}_{\Psi} s\left(a_{k}, \cdot\right)\right|\right)\right|, \quad k=0,1, \ldots, 100
$$

which is nothing but an application of the envelope method to the restriction of the CWT to frequency $\omega_{0} / a_{k}$ (the use of a complex-valued Morlet wavelet eliminates the need for demodulation).

Hence, if there are some $k_{1}, k_{2}$ and $n$ such that $e_{k}$ has maximum amplitude $e_{k}(n)$ for $k=k_{1}, k_{1}+1, \ldots, k_{2}$ and such that $\sum_{k=k_{1}}^{k_{2}} e_{k}(n) / \sum_{k=0}^{100} e_{k}(n)$ is "close enough" to 1 , then one can expect the analyzed signal $s$ to contain some transient and periodically repeating oscillations with oscillation frequency $\approx$ $\omega_{0} / a_{k} \mathrm{~Hz}$ and repetition frequency $\approx n f_{\mathrm{s}} / N \mathrm{~Hz}$, where $k_{1} \leq k \leq k_{2}$ and, where $N$ is the length (number of samples) of $s$. This is the intuitive motivation for how the CWT2-algorithm automatically chooses the oscillation frequencies with largest SNR in measurements on defect bearings, and finds the "most dominating" repetition frequencies at those oscillation frequencies. We begin, however, with the simpler and less adaptive CWT1 algorithm.

CWT1: Figure 12 (a) contains example plots of $\sum_{k=0}^{100} e_{k}$ for the signals I3 and I6 (from a worm screw pump before and after replacement of a bearing with a large pitting damage with material flaking). For the automatic classification in figures 12 (b) and (c) we have used the following algorithm:

(1) For each repetition frequency in (3) and each measurement direction,

(a) compute $\sum_{k=0}^{100} e_{k}$ with $e_{k}$ defined in (11).

(b) Apply the same automatic evaluation as for the envelope method in Section 5.1 but keep only the largest peak-to-median ratio.

(2) Choose the two largest peak-to-median ratios computed in step (1).

CWT2: In Figure 13 (a) we have applied the basic steps of the method CWT2 to signal H2 (from a drying cylinder bearing with unevenly distributed pits in the outer race): For each $e_{k}$ in (11), we have plotted the amplitude and the associated repetition frequency $n f_{\mathrm{s}} / N$ of the 3 largest peaks in the topmost two plots. For every point at the $y$-axis of the second plot, we have then checked at which oscillation frequencies (if any) this is the dominating repetition frequency, summed those peak amplitudes, divided with the sum of all peak values and plotted the result in subplot 3 . Then we repeated the last step for the two other curves in the second plot to get the lowermost two plots. This type of figures gives a rather good overview of the dominating repetition frequencies at different oscillation frequencies. For example, we can clearly see both the outer ring frequency $24 \mathrm{~Hz}$ and several of its harmonics.

The full algorithm for finding potentially interesting $k \mathrm{~s}$, corresponding repetition frequencies and producing the classification plots in Figure 13 (b), goes as follows: 
(1) For each measurement direction and for each repetition frequency $1 / T$ in (3), do the following.

(a) Apply a wavelet packet noise reduction with Daubechies 9 wavelet, nine levels expansion and keeping the $10 \%$ largest coefficients (see, e.g., $[27,28])$.

(b) For $n=1,2, \ldots, N$ and for $m=1,2,3$, let $K_{m}$ be the set of integers $k$ such that $e_{k}(n)$ is the amplitude of the $m$ th largest peak and set
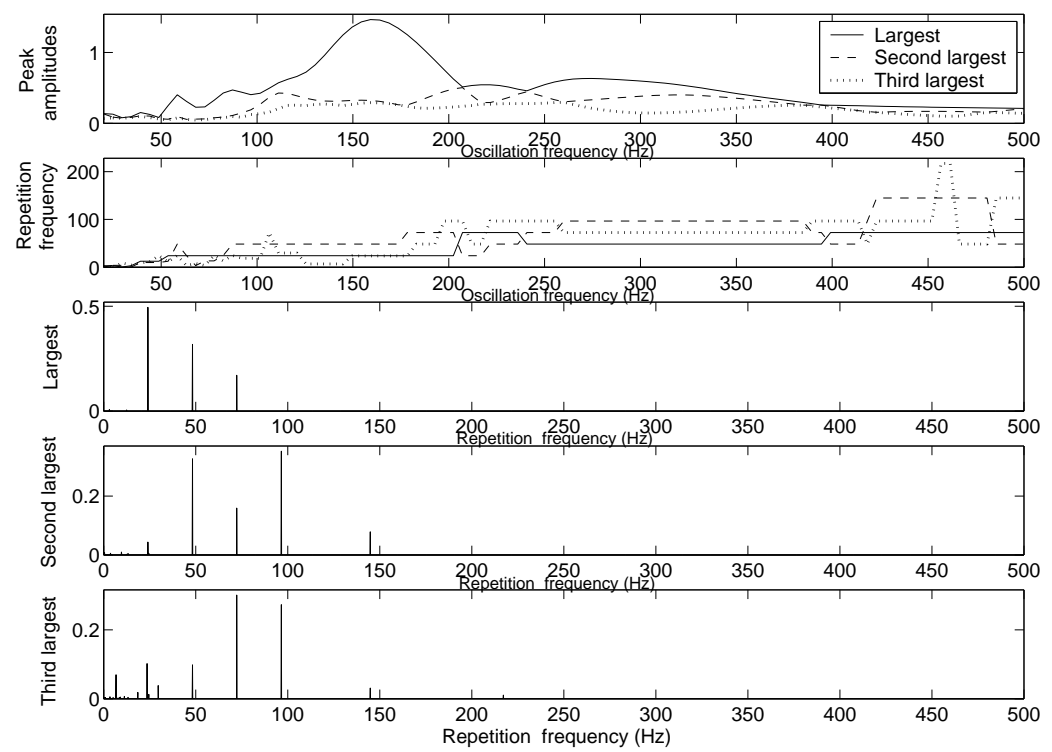

(a)

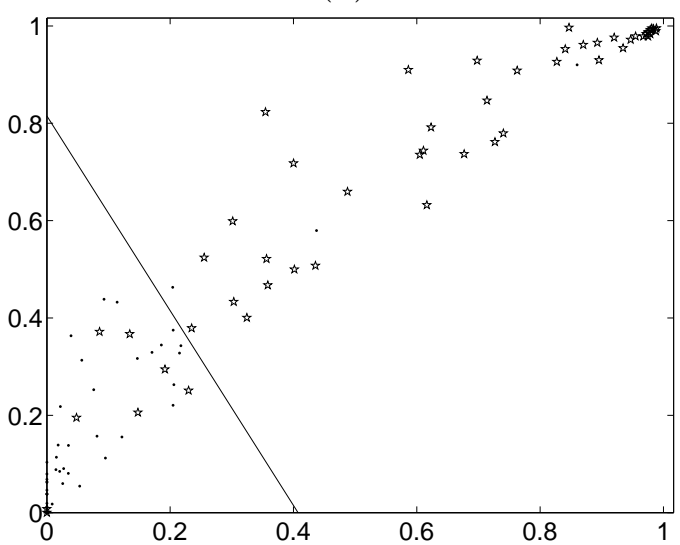

(b)

Fig. 13. CWT2 method example plot for the signal H2: (a) The topmost two subplots show the amplitude and repetition frequency $n f_{\mathrm{s}} / N$ (plotted as functions of the oscillation frequency $\omega_{0} / a_{k}$ ) of the largest, second largest and third largest peak of each $e_{k}$. The remaining subplots show the associated relative peak amplitudes (computed as described in the text) at each repetition frequency. (b) Automatic classification $(\cdot=$ functional and $\star=$ defect bearing $)$. The misclassification rate is $11 / 103 \approx 10.7 \%$. 


$$
S_{m, n}=\sum_{k \in K_{m}} e_{k}(n) / \sum_{k=1}^{100} e_{k}(n) .
$$

(c) Let $M$ be the set of integers $n$ such that $n f_{\mathrm{s}} / N$ is in some neighborhood (depending on with how good precision $T$ is known) of $1 / T$, $2 / T$ or $3 / T$. For $m=1,2,3$, compute $\sum_{n \in M} S_{m, n}$.

(2) From all computations of the sum on the previous line, keep the two largest values.

\subsection{Summary of experimental results}

Table 2 is a summary of the registered misclassifications for our best performing methods. We describe different possible causes for these misclassifications in [19]. The most frequent suggestions are low signal to noise ratio (SNR) and/or an impact frequency that coincides with twice the feeding frequency of the motor.

\begin{tabular}{|c|c|c|c|c|}
\hline Miss & Envelope & Periodization & CWT 1 & CWT 2 \\
\hline $\mathrm{C} 1$ & & • & & $\bullet$ \\
\hline F1 & $\bullet$ & & $\bullet$ & \\
\hline $\mathrm{F} 2$ & • & & $\bullet$ & • \\
\hline F3 & $\bullet$ & & $\bullet$ & \\
\hline $\mathrm{F} 4$ & $\bullet$ & & $\bullet$ & $\bullet$ \\
\hline H5 & & & $\bullet$ & \\
\hline $\mathrm{K} 1 \mathrm{~b}$ & & $\bullet$ & & \\
\hline L2a & - & & $\bullet$ & $\bullet$ \\
\hline N1a & $\bullet$ & $\bullet$ & $\bullet$ & $\bullet$ \\
\hline $\mathrm{N} 1 \mathrm{~b}$ & $\bullet$ & $\bullet$ & & $\bullet$ \\
\hline $\mathrm{N} 2 \mathrm{a}$ & & & & $\bullet$ \\
\hline $\mathrm{N} 2 \mathrm{~b}$ & & • & & $\bullet$ \\
\hline \multicolumn{5}{|l|}{ False alarm } \\
\hline A1 & $\bullet$ & • & $\bullet$ & $\bullet$ \\
\hline $\mathrm{A} 2$ & • & $\bullet$ & & \\
\hline A3 & & $\bullet$ & & \\
\hline B5 & & $\bullet$ & $\bullet$ & \\
\hline $\mathrm{B} 6$ & & $\bullet$ & & \\
\hline D4 & & $\bullet$ & & \\
\hline E6 & & & & - \\
\hline F7 & & $\bullet$ & & \\
\hline I6 & & & & $\bullet$ \\
\hline M1 & - & & & \\
\hline Misclassification rate & $\frac{10}{103} \approx 10 \%$ & $\frac{12}{89} \approx 13 \%$ & $\frac{9}{103} \approx 9 \%$ & $\frac{11}{103} \approx 11 \%$ \\
\hline
\end{tabular}

Table 2

For the methods with lowest misclassification ratios, all misclassification ocurred for the above 22 (of 103) test signals (described in detail in in [19, p. 58-64]). 
We have chosen test signals for which all bearing defects are are clearly visible and described in more detail in [19, p. 58-64]. For those sharp defects that were detected in plots like Figure 13 (b), the plotted points are generally more far away from the classification line for defects in otherwise smooth inner and outer ring surfaces (as expected in the case of model deviations or lower SNR).

Most methods (with periodization as a notable exception) have some problems with signals F1-4 (see Table 2), which are measured on a slowly rotating (93 rpm) drying cylinder with an outer race bearing defect. Here we think that one important reason for the high miss rate is a low SNR caused by the fact that all signals are are bandpass filtered with the same bandpass filter and a lower cutoff-frequency $150 \mathrm{~Hz}$, which is very close to and interfers with the oscillation frequency.

We note also that although our methods primarily were designed for welllocalized single defects, some unevenly distributed defects, like signal O1-3 (large pitting damage and additional axial cracks along the entire raceway) and H1-4 (unevenly distributed damages), were correctly detected, by all methods. Still, some others (like F1-4, which have visible fatigue damages with pitting on $1 / 3$ of the circumference of the outer race) was not.

So, altogether the above observations indicate a combination of both model dependence and robustness against model errors. Still, the misclassification rates in Table 2 show that, at this point, there is more or less an even race between these four methods, so we consider it worthwhile to continue refining all of them, for example according to our suggestions in next section.

\section{Conclusions and suggested improvements}

From a theoretical point of view, we concluded that wavelet-techniques are particularly well suited for bearing monitoring methods that makes use of our signal and noise model but without being too sensitive to inevitable deviations from this model (due to the big variety of different machines and industrial environments).

From our experimental results, we conclude that our four best performing methods (and some more) all are good candidates for further refinements towards really good methods. Right now, they are already fully automatic, there is a lot of thought behind all considered methods, some trial and error with test signals for improvements and then our large systematic test to rule out the four currently best ones. Still, we have chosen to test many (combinations of) methods rather than choosing one or a few and really start optimizing. Hence we want to point out both some recommended improvements and a few 
more variations and alternatives that could deserve some closer study:

\section{Suggested improvements}

Trend charts recall from Section 3 that our methods only analyze the shape of the signal. Hence, the probably most evident route to improved performance is to also use historical data, trending (see, e.g., $[2,1]$ ) or comparison with some other known reference values.

Classification line: We only have one set of test signals both for choosing optimal classification line and for the classification. For more decisive evaluations, it would be better to use two separate and large sets of representative and well-classified test signals. Moreover, although our classification lines in $\mathbb{R}^{2}$ are practical for visual demonstratios, for optimal performance, one should probably use some other $\mathbb{R}^{n}$ and a standard classification method, such as linear discriminant analysis [31]. (For bearing monitoring, or related problems, classification methods based on pattern recognition, artificial neural network and expert system based on fuzzy logic have also been used, see e.g. $[32,11,33,34,15,16,35]$.)

Adapt methods to signal and environment characteristics: Contrary to the classification line, our methods were developed and tested on another (much smaller) set of test functions. Then they were used without changes on our test signals, instead of adapting crucial parts like as deconvolution bandpass filters to usually known signal characteristics, such as in the following concrete examples:

- Improve model correctness by adjusting bandpass filters to block frequencies where model deviations or disturbing vibrattions are known to exist (as described in the bullet list in Section 2.4) but not too close to the bearing impact oscillation frequency (as for signals F1-4 in Section 5.4).

- Another example (mentioned in Section 5.4) is some misclassifications of functional bearings for which twice the roller frequency coincided with twice the motor feeding frequency. This is a common vibration frequency in $\mathrm{AC}$ motors but defect rollers are very unusual, so by not checking for roller defects, one should get lower false alarm rate without a corresponding increase of the miss rate.

Adapt measurement parameters to method and environment characteristics: We have used test signals with sampling frequencies ranging from 512 to $25600 \mathrm{~Hz}$ and with signal lengths 4096-16384 samples. These parameters were not optimized for any particular method or important system parameters (such as resonance frequency or impact frequency). This is sometimes unavoidable (e.g. depending on available measurement equipment) but whenever possible, we recommend a more careful choice for each environment (or other solutions, such as the upsampling in the envelope method). See (2) below for a concrete example.

Use full wavelet packet tree instead of just the nine levels used in the 
envelope and CWT2 methods (for time-saving reasons).

Parameter choices etc: Especially for the wavelet based methods there are loads of parameters, settings and small choices for which a whole lot of thought and experimenting may be needed for finding (in some sense) optimal combinations. For instance, it can be thresholds, entropies, choice of wavelet, how to choose scaling parameters $a_{k}$ in (10), how they should be summed (e.g., weighted sums or $l_{p}-s u m$ ) or if the fact that we have synchronized measurements in three orthogonal directions can be exploited in better ways.

\section{Some other potentially interesting methods and variations}

Different "periodicity measures": We have used either periodization or the FFT as core method for finding impact periodicites in all our methods (although in combination with different other tools in different methods). For this step other variants, such as the following examples, could be worth some testing:

- Use the periodization method but compute the maximum-to-minimum ratio instead of maximum in step 1 of the algorithm (but then without subtracting the mean value, as done by the bandpass filter there).

- One more alternative is to study the matrix whose $n$th row contain the sample values corresponding to the interval $[(n-1) T, n T)$ for $n=1,2, \ldots, N$. The quotient between the two largest singular values of this matrix then reflects how "close to periodic" the signal is (see [36]).

The LDB algorithm (see Section 3.5) is so far only evaluated for a small number of test signals, but deserves a more careful test.

A refined signal model may (or may not) make the tested statistical methods more useful (see sections 2.2, 2.4 and 3.4).

Decrease input SNR As described after (9), SNR is, by matched filter theory, minimized if the input $a=s^{\prime \prime}$ is replaced with a certain linear combination of $s, s^{\prime}$ and $s^{\prime \prime}$. As described there, we found no clear improvement of SNR in our simple test, but still, a closer study (possibly in combination with a refined signal model or other improvements described above) may very well lead to large enough SNR improvements to be interesting either in general or for some particular type of environments.

Finally, we summarize our experiences from this project in three basic rules of thumb for developing and applying a good condition monitoring method:

(1) Collect any available and relevant data about the system at hand. For example, is it possible to estimate the impulse response? Does any other source of vibration coincide with the impact frequencies the method is designed to alarm for?

(2) Given the memory size of the measurement device, choose sampling fre- 
quency with care. It must be large enough for the algorithm to capture the basic resonance frequency, but also small enough to give long enough measurements to capture several impacts.

(3) As far as possible, try to adapt all parameters associated with the method for optimal performance with the system and measurement properties described in 1 and 2 .

\section{References}

[1] G. White, Introduction to Machine Vibration, DLI Engineering, 1994, WWW: http://www.dliengineering.com/section.asp?nID=19.

[2] V. Wowk, Machinery Vibration: Measurement and Analysis, McGraw-Hill, Inc., N. Y., 1991.

[3] S. Braun, The signature analysis of sonic bearing vibration, IEEE Transactions on Sonics and Ultrasonics (SU-27) (1980) 317-328.

[4] S. Braun, B. Datner, Analysis of roller/ball bearing vibrations, Journal of Mechanical Design (101) (1979) 118-125.

[5] D. Dyer, R. Stewart, Analysis of rolling element damage by statistical vibration analysis, Journal of Mechanical Design (100) (1978) 229-235.

[6] D. Logan, J. Mathew, Using the correlation dimension for vibration fault diagnosis of rolling element bearing-I. Basic concepts, Mechanical Systems and Signal Processing (10) (1996) 241-250.

[7] P. McFadden, J. Smith, Model for the vibration produced by a singel point defect in a rolling element bearing, Journal of Sound and Vibration (96) (1984) $69-82$.

[8] J. Shiroishi, Y. Li, S. Liang, T. Kurfess, S. Danyluk, Bearing condition diagnosis via vibration and acoustic emission measurements, Mechanical Systems and Signal Processing (11) (1997) 693-705.

[9] J. Taylor, Identification of bearing defects be spectral analysis, Journal of Mechanical Design (102) (1980) 199-204.

[10] Y. Wang, P. Kootsookos, Modeling of low shaft speed bearing faults for condition monitoring, Mechanical Systems and Signal Processing (12) (1998) $415-426$.

[11] H. Choe, Y. Wan, A. Chan, Neural pattern identification of railroad wheelbearing faults from audible acoustic signals: Comparison of FFT, CWT, and DWT features, Proceedings of the SPIE (3078) (1997) 480-496.

[12] G. Dalpiaz, A. Rivola, Condition monitoring and diagnostics in automatic machines: Comparison of vibration analysis techniques, Mechanical Systems and Signal Processing (11) (1997) 53-73. 
[13] M. Ladd, G. Wilson, Proportional bandwith properties of fault indicating tones in a ball bearing system, IEEE Comput. Soc. Press (1994) 45-49.

[14] C. J. Li, J. Ma, Bearing localized defect detection through wavelet decomposition of vibration, in: Sensors and Signal Processing for Manufacturing (Proc. of Winter Annual Meeting of the American Society of Mechanical Engineers), Vol. 55 of ASME Prod Eng Div Publ PED, ASME, New York, NY, USA, Anaheim, CA, USA, 1992, pp. 187-196.

[15] B. Paya, I. Esat, M. Badi, Artificial neural network based fault diagnostics of rotating machinary using wavelet transforms as a preprosessor, Mechanical Systems and Signal Processing (11) (1997) 751-765.

[16] W. Staszewski, G. Tomlinson, Application of the wavelet transform to fault detection in a spur gear, Mechanical Systems and Signal Processing (8) (1994) 289-307.

[17] W. Wang, P. McFadden, Application of orthogonal wavelets to early gear damage detection, Mechanical Systems and Signal Processing (9) (1995) 497507.

[18] Y. Wu, R. Du, Feature extraction and assement using wavelet packets for monitoring of machining processes, Mechanical Systems and Signal Processing (10) (1996) 29-53.

[19] S. Ericsson, N. Grip, E. Johansson, L.-E. Persson, R. Sjöberg, J.O. Strömberg, Automatic detection of local bearing defects in rotating machines - Part I, Research report 2002 - 16 (69 pages), Department of Mathematics, Luleå University of Technology, SE-971 87 Luleå, WWW: http://www.sm.luth.se/ grip/Research/publications.html (Sep. 2001).

[20] T. A. Harris, Rolling Bearing Analysis, 4th Edition, John Wiley \& Sons, Inc, 2001.

[21] J. L. Meriam, L. Kraige, Dynamics, 2nd Edition, Vol. 2, John Wiley and Sons, 1987.

[22] A. V. Oppenheim, R. W. Schafer, Discrete-time signal processing, Prentice Hall, NJ, USA, 1989.

[23] A. Papoulis, S. U. Pillai, Probability, Random Variables and Stochastic Processes, 4th Edition, McGraw-Hill, 2001.

[24] R. B. Randall, Frequency analysis, 3rd Edition, Brüel \& Kjærr, Nærum, Denmark, 1987.

[25] I. Daubechies, Ten Lectures on Wavelets, SIAM, Philadelphia, Pennsylvania, 1992.

[26] N. Grip, Wavelet and Gabor frames and bases: Approximation, sampling and applications, Doctoral thesis 2002:49, Luleå University of Technology, SE-971 87 Luleå,

WWW: http://www.sm.luth.se/ grip/Research/publications.html (2002). 
[27] J. Bergh, F. Ekstedt, M. Lindberg, Wavelets, Studentlitteratur, Lund, Sweden, 1999.

[28] S. Mallat, A wavelet tour of signal processing, 2nd Edition, Academic Press, London, 1999.

[29] K. Gröchenig, Foundations of Time-Frequency Analysis, Birkhäuser, 2000.

[30] E. Johansson, Faulty bearing detection with wavelet feature extraction, Masters thesis / 2000:268, Luleå University of Technology, SE-971 87 Luleå (Oct. 2000).

[31] K. Fukunaga, Introduction to statistical pattern recognition, 2nd Edition, Academic Press, Boston, 1990.

[32] D. Baillie, J. Mathew, A comparison of autoregressive modeling techniques for fault diagnosis of rolling element bearings, Mechanical Systems and Signal Processing (10) (1996) 1-17.

[33] T. Liu, J. Mengel, Intelligent monitoring of ball bearing conditions, Mechanical Systems and Signal Processing (6) (1992) 419-431.

[34] T. Liu, J. Singonahalli, N. Iyer, Detection of roller bearing defects using expert system and fuzzy logic, Mechanical Systems and Signal Processing (10) (1996) 595-614.

[35] W. Staszewski, K. Worden, G. Tomlinson, Time-frequency analysis in gearbox fault detection using Wigner-Ville distribution and pattern recognition, Mechanical Systems and Signal Processing (11) (1997) 673-692.

[36] P. Kanjilal, S. Palit, On multiple pattern extraction using singular value decomposition, IEEE Transactions on Signal Processing 43 (6) (1995) 15361540. 